\title{
Tsallis statistics and generalized uncertainty principle
}

\author{
Giuseppe Gaetano Luciano ${ }^{1,2, a_{0}}$ \\ ${ }^{1}$ Dipartimento di Fisica, Università di Salerno, Via Giovanni Paolo II, 132, 84084 Fisciano, SA, Italy \\ ${ }^{2}$ INFN, Sezione di Napoli, Gruppo Collegato di Salerno, Via Giovanni Paolo II, 132, 84084 Fisciano, SA, Italy
}

Received: 4 June 2021 / Accepted: 25 July 2021 / Published online: 30 July 2021

(C) The Author(s) 2021

\begin{abstract}
It has been argued that non-Gaussian statistics provide a natural framework to investigate semiclassical effects in the context of Planck-scale deformations of the Heisenberg uncertainty relation. Here we substantiate this point by considering the Unruh effect as a specific playground. By working in the realm of quantum field theory, we reformulate the derivation of the modified Unruh effect from the generalized uncertainty principle (GUP) in the language of the nonextensive Tsallis thermostatistics. We find a nontrivial monotonic relation between the nonextensivity index $q$ and the GUP deformation parameter $\beta$, which generalizes an earlier result obtained in quantum mechanics. We then extend our analysis to black hole thermodynamics. We preliminarily discuss our outcome in the broader context of an effective description of Planck-scale gravitational physics based on Tsallis theory.
\end{abstract}

\section{Introduction}

In the last decades several models of quantum gravity, such as String Theory, Loop Quantum Gravity, Quantum Geometry and Doubly Special Relativity, and heuristic arguments on black hole physics have converged on the idea that the Heisenberg uncertainty principle (HUP) should be amended at Planck scale to account for the emergence of a minimal measurable length [1-14]. The ensuing uncertainty relations are known as Generalized Uncertainty Principles (GUP's). Among the various deformations proposed in literature, a paradigmatic form of GUP is the quadratic GUP

$\Delta x \Delta p \geq \frac{1}{2}\left[1+\beta\left(\frac{\Delta p}{m_{p}}\right)^{2}\right]$,

where we have set the speed of light $c$ and the Planck constant $\hbar$ equal to unity. Here $m_{p} \simeq 10^{-8} \mathrm{~kg}$ is the Planck mass, although in a broader sense it may represent any other inter-

a e-mail: gluciano@unisa.it (corresponding author) mediate mass scale present in the theory [15]. For instance, in the case of lattice systems and for momenta deep inside the Brillouin zone, it turns out to be related to the inverse lattice spacing. The (dimensionless) deformation parameter $\beta$ quantifies the departure of the GUP (1) from the HUP. In principle, it is not fixed by the theory, despite being generally taken of order unity in some models of string theory [1-4]. Nevertheless, many studies are being developed to constrain $\beta$ by using either theoretical arguments [15-24] or experimental tests [25-31] (see Ref. [32] for a recent review). Concerning the sign of the GUP correction, it is straightforward to check that Eq. (1) leads to a minimal position uncertainty of the order of Planck length $\ell_{p}=1 /\left(2 m_{p}\right) \simeq 10^{-35} \mathrm{~m}$ for $\beta>0$, while no length cutoff emerges for $\beta<0$ [7,8]. Henceforth, we shall focus on the $\beta$-positive case. Clearly, the standard Heisenberg relation is recovered for $\beta \rightarrow 0$.

For mirror-symmetric states (i.e. $\langle p\rangle=0$ ), the relation (1) is equivalent to the modified commutator

$$
[x, p]=i\left[1+\beta\left(\frac{p}{m_{p}}\right)^{2}\right],
$$

because of Robertson's inequality $\Delta x \Delta p \geq 1 / 2|\langle[x, p]\rangle|$. Vice-versa, the commutator (2) implies Eq. (1) for any state. To simplify the notation, here and henceforth we omit the symbol ^ over the quantum operators when no ambiguity arises.

As a consequence of the deformed commutator between $x$ and $p$, also the phase space structure gets significantly affected. Indeed, as shown in [33], the recipe (1) yields a modification of the elementary cell volume occupied by each quantum state of the system. Implications of the minimumlength deformed quantum mechanics on a statistical level have been considered in a variety of contexts, ranging from the computation of the GUP correction to the black-body radiation [34-36], to the study of novel condensation phenomena in Maxwell-Boltzmann statistics [37] and the cosmological problem [33]. Recently, deep investigation has also been carried out within the framework of the thermo- 
dynamics of the ideal gas $[38,39]$ and a general scheme to calculate the quantum partition function has been developed in [40] for various systems.

All of the above studies suggest that the quantum statistical mechanics should be somehow reformulated when including the GUP. Preliminary efforts to establish a link between a given statistical framework and the ensuing modification of the phase space cell volume have been done in [41-45]. Specifically, in $[42,45]$ the question arose as to how the phase space structure would appear in the context of Tsallis statistics based on a nonadditive redefinition of the entropy [4649]. We recall that Tsallis theory is a nonextensive generalization of Boltzmann-Gibbs thermodynamics parameterized by the entropic index $q$, the departure from extensivity being quantified by $|q-1|$ (in other terms, $q \neq 1$ corresponds to the non-Gaussian regime, while $q \rightarrow 1$ gives back the standard Boltzmann-Gibbs theory). The concept was introduced as a basis for extending the traditional statistical mechanics and is formally identical to Havrda-Charvát structural $\alpha$-entropy considered in the information theory [50]. On the other hand, the reverse problem of seeking for the statistics that emerges from the modification of the phase space cell volume implied by the GUP has been addressed in [51]. In particular, it has been shown that generalized statistics with a quadratic correction over Gaussian profile arise quite naturally if one assumes a change in the elementary cell volume as prescribed by the GUP (1), under the condition that the total phase space volume is kept invariant. The correspondence between the two frameworks has been investigated concretely in the contexts of Unruh effect [52] and Jeans instability [53], proving that quadratic non-Gaussian statistics produce the same effects as the GUP (1) at the level of the modified Unruh temperature and Jeans mass, respectively. In turn, this allows establishing a link between the GUP parameter $\beta$ and the index $q$ that underlies the modified statistics [51].

Starting from the above premises, here we analyze the connection between non-Gaussian statistics and the GUP by considering the Unruh effect as a specific playground. We focus on the generalized statistics arising from Tsallis's nonextensive prescription, which is known to affect the core thermostatistic relations, including the zero-th law of thermodynamics and the Maxwell-Boltzmann, Fermi-Dirac and BoseEinstein distributions [54-58]. For our purposes, we exploit the result of [59], where the Unruh effect has been studied in the context of quantum field theory with a quadratic GUP, obtaining a $\beta$-dependent expression for the particle spectrum of the vacuum condensate. It is important to stress that our approach is conceptually different from that in [51]. Indeed, the latter relies on the comparison between the GUP- and Tsallis-induced corrections at the level of the phase space cell volume and the ensuing effects on the energy/temperature relation. By contrast, we set out the conditions under which the consistency is obtained at the level of the generalized distribution of Unruh vacuum condensate. In doing so, we come up with a nontrivial monotonic relation between the parameters $\beta$ and $q$. We discuss our result compared to the correspondence found in [51]. We then extend our considerations to black hole thermodynamics. In this context, we deal with the computation of the modified black hole temperature and entropy based on both the GUP and Tsallis approaches.

The layout of the paper is as follows. In the next section we briefly review the derivation of Unruh effect in both the canonical and GUP frameworks. To this end, we closely follow [59]. Section 3 is devoted to the comparison with the $q$ generalized Tsallis statistics. A similar analysis is developed in the final Appendix in the context of black hole thermodynamics. Conclusions and outlook are finally discussed in Sect. 4.

\section{Unruh effect from Heisenberg and generalized uncertainty principles}

The Unruh effect predicts that a uniformly accelerated (Rindler) observer measures a Planck emission distribution in the vacuum state of inertial observers, the temperature $T_{\mathrm{U}}$ being proportional to the magnitude of his acceleration. Since its original formulation based on Bogoliubov transformation approach [52], this result has been re-derived by different techniques, such as the Doppler-shift method [60] or the Unruh-DeWitt detector model [61], thus proving to be one of the most firmly rooted predictions of quantum field theory.

In what follows, we rely on the Bogoliubov transformation approach. Toward this end, we first review the canonical quantization of the scalar field in Minkowski spacetime. Then, we introduce the so-called Rindler-Fulling quantization scheme, which generalizes the above formalism to the Rindler metric. We show that the comparison between the two field expansions naturally leads to the emergence of the Unruh effect as explained above. To avoid unnecessary technicalities, we consider a neutral scalar field of mass $m$ in 1+1-dimensions. In addition, we use the Minkowski metric with the conventional timelike signature

$d s^{2}=\eta_{\mu \nu} d x^{\mu} d x^{\nu}=d t^{2}-d x^{2}$.

\subsection{Canonical field quantization}

In Minkowski spacetime the scalar field in the standard planewave representation reads ${ }^{1}$

$\Phi(\mathbf{x})=\int d k\left[a_{k} U_{k}(\mathbf{x})+a_{k}^{\dagger} U_{k}^{*}(\mathbf{x})\right]$,

\footnotetext{
${ }^{1}$ In what follows, the words Minkowski and inertial are taken as interchangeable.
} 
where we have denoted by $\mathbf{x} \equiv\{t, x\}$ the set of Minkowski coordinates. Here, $U_{k}$ is the (normalized) planewave of momentum $k$ and positive frequency $\omega_{k}=\sqrt{m^{2}+k^{2}}$, defined by

$U_{k}(\mathbf{x})=\left(4 \pi \omega_{k}\right)^{-\frac{1}{2}} e^{i\left(k x-\omega_{k} t\right)}$.

In the canonical second quantization formalism, the expansion coefficients $a_{k}$ and $a_{k}^{\dagger}$ are promoted to operators satisfying the commutation relation

$\left[a_{k}, a_{k^{\prime}}^{\dagger}\right]=\delta\left(k-k^{\prime}\right)$,

with all other commutators vanishing. They play the rôle of annihilation and creation operators of Minkowski quanta. The Minkowski vacuum $\left|0_{\mathrm{M}}\right\rangle$ is accordingly defined by $a_{k}\left|0_{\mathrm{M}}\right\rangle=0, \forall k$.

A comment is now in order: as well known, in the more general four-dimensional case, the Poincaré group of symmetries of Minkowski spacetime is ten-dimensional, containing four spacetime translations, three spatial rotations and three spatio-temporal rotations, i.e. the Lorentz boosts. The expansion (4) is associated to the first symmetry, being the planewaves eigenmodes of the energy-momentum operator. To make it easier the extension of the field quantization formalism to the Rindler framework, we exploit the symmetry under Lorentz boosts and introduce the so-called hyperbolic (or boost-mode) spectral representation $[62,63]$

$\Phi(\mathbf{x})=\int_{0}^{+\infty} d \Omega \sum_{\sigma}\left[d_{\Omega}^{(\sigma)} \widetilde{U}_{\Omega}^{(\sigma)}(\mathbf{x})+d_{\Omega}^{(\sigma) \dagger} \widetilde{U}_{\Omega}^{(\sigma) *}(\mathbf{x})\right]$,

where the modes $\widetilde{U}_{\Omega}^{(\sigma)}$ are eigenfunctions of the Lorentz boost along the $x$-axis. They are given by linear combinations of positive-frequency planewaves only, i.e.

$\widetilde{U}_{\Omega}^{(\sigma)}(\mathbf{x})=\int d k p_{\Omega}^{(\sigma) *}(k) U_{k}(\mathbf{x})$,

where

$p_{\Omega}^{(\sigma)}(k)=\frac{1}{\sqrt{2 \pi \omega_{k}}}\left(\frac{\omega_{k}+k}{\omega_{k}-k}\right)^{i \sigma \Omega / 2}$,

with $\sigma= \pm$ and $0<\Omega<\infty$. The physical meaning of such quantum numbers will be clarified soon.

It is easy to verify that the quantizations (4) and (7) are completely equivalent from the point of view of the associated Fock spaces of number states. Indeed, a straightforward substitution of Eq. (8) into (7) yields

$d_{\Omega}^{(\sigma)}=\int d k p_{\Omega}^{(\sigma)}(k) a_{k}$,

which allows establishing that $d_{\Omega}^{(\sigma)}\left|0_{\mathrm{M}}\right\rangle=0$, i.e. $\left|0_{\mathrm{M}}\right\rangle$ is still vacuum for the $d$-operators. This could be somehow expected, since each mode $\widetilde{U}_{\Omega}^{(\sigma)}$ is a superposition of positivefrequency planewaves only. Furthermore, the completeness

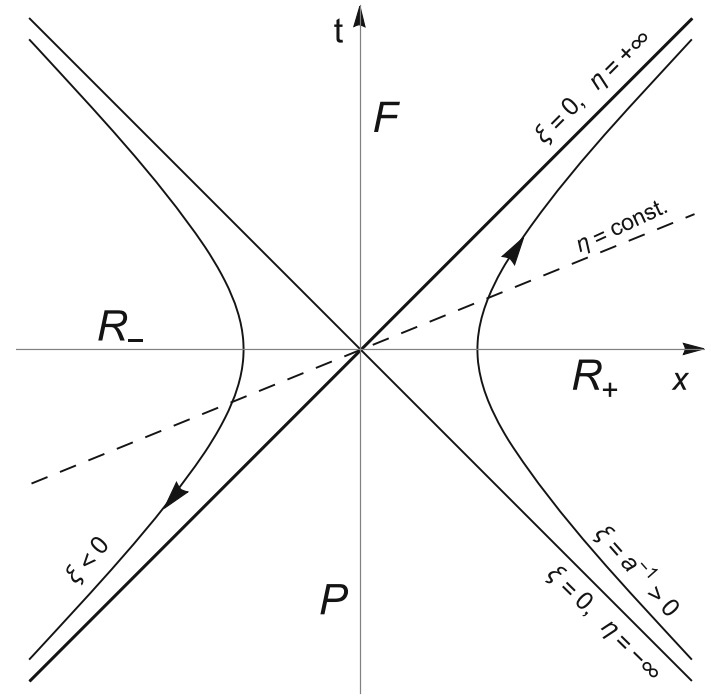

Fig. 1 Rindler chart of a uniformly accelerated (Rindler) observer in Minkowski spacetime

and orthonormality of the set of functions $\left\{p_{\Omega}^{(\sigma)}\right\}$ guarantee that the transformation (10) is canonical [64], i.e.

$\left[d_{\Omega}^{(\sigma)}, d_{\Omega^{\prime}}^{\left(\sigma^{\prime}\right) \dagger}\right]=\delta_{\sigma \sigma^{\prime}} \delta\left(\Omega-\Omega^{\prime}\right)$,

which completes the proof of the equivalence.

We are now ready to describe the Rindler-Fulling scheme. This is the most natural quantization to adopt for an uniformly accelerated observer moving through Minkowski space. Moreover, it provides a paradigmatic example of the problem of the unitarily inequivalent representations arising in quantum field theory on curved spacetime [65]. As a first step, we introduce the Rindler coordinates $\{\eta, \xi\}$, which are related to $t$ and $x$ by

$t=\xi \sinh \eta, \quad x=\xi \cosh \eta$,

with $-\infty<\eta, \xi<\infty$. In terms of these coordinates, the line element takes the form

$d s^{2}=\xi^{2} d \eta^{2}-d \xi^{2}$,

which admits $B=\partial_{\eta}$ as a timelike Killing vector.

Strictly speaking, the chart (12) only covers two regions of Minkowski spacetime, namely the right wedge $R_{+}=$ $\{\mathbf{x}|x>| t \mid\}$ for $\xi>0$, and the left wedge $R_{-}=$ $\{\mathbf{x}|x<-| t \mid\}$ for $\xi<0$ wedge (see Fig. 1). In the remaining sectors $F$ and $P$, the rôles of space and time coordinates are reversed, so that one has $t=\xi \cosh \eta$ and $x=\xi \sinh \eta$, with $\xi>0(\xi<0)$ in $F(P)$.

From considerations of relativistic kinematics, the worldline of a uniformly accelerated observer with proper acceleration $a>0$ is given in Rindler coordinates by $\xi(\tau)=$ const $=a^{-1}$, where $\tau=\eta / a$ denotes the proper time along the trajectory. In terms of Minkowski coordinates, this is a 
branch of hyperbola of null asymptotes $x= \pm t$ (i.e. $\xi=0$ ). The hypersurfaces $\eta=$ const describe simultaneous events from the point of view of such an observer. Remarkably, the Rindler manifold for $\xi>0$ cannot be extended to $\xi<0$ through the hypersurface $\xi=0$. This indeed represents an event horizon for the Rindler observer, which cannot access any event located beyond it (in particular, the upper (lower) hypersurface $\xi=0, \eta=+\infty(\xi=0, \eta=-\infty)$ acts as a future (past) event horizon). In passing, we note that the above features of Rindler and Minkowski metrics are analogous to that of Schwarzschild spacetime inside the Kruskal one, from both the geometrical and physical point of view.

The peculiar causal structure of Rindler metric nontrivially affects the field quantization. Since the two wedges $R_{ \pm}$are causally disconnected from each other, the positivefrequency solutions of Klein-Gordon equation in Rindler coordinates must be written as [64]

$u_{\Omega}^{(\sigma)}(\mathbf{x})=N_{\Omega} \theta(\sigma \xi) K_{i \Omega}^{(\sigma)}(m \xi) e^{-i \sigma \Omega \eta}$,

where the $\theta$-Heaviside function restricts the domain of $u_{\Omega}^{(\sigma)}$ to either $R_{+}$(for $\sigma=+$ ) or $R_{-}$(for $\sigma=-$ ). With an abuse of notation, here we have used the symbol $\mathbf{x}$ for the Rindler coordinates as well. The positive parameter $\Omega$ plays the rôle of frequency respect to the Rindler time $\eta$. The coefficient $N_{\Omega}$ can be fixed by requiring that the above modes are normalized with respect to the inner product in Rindler coordinates [64]. In addition, we notice that the spatial dependence is only contained in the modified Bessel function $K_{i \Omega}^{(\sigma)}$. We shall refer to the modes (14) as Rindler modes.

Within the above framework, the field expansion for the Rindler observer reads

$\phi(\mathbf{x})=\int_{0}^{+\infty} d \Omega \sum_{\sigma}\left[b_{\Omega}^{(\sigma)} u_{\Omega}^{(\sigma)}(\mathbf{x})+b_{\Omega}^{(\sigma) \dagger} u_{\Omega}^{(\sigma) *}(\mathbf{x})\right]$.

The ladder operators $b_{\Omega}^{(\sigma)}$ and $b_{\Omega}^{(\sigma) \dagger}$ are still assumed to obey the canonical commutation relations

$\left[b_{\Omega}^{(\sigma)}, b_{\Omega^{\prime}}^{\left(\sigma^{\prime}\right) \dagger}\right]=\delta_{\sigma \sigma^{\prime}} \delta\left(\Omega-\Omega^{\prime}\right)$,

with all other commutators vanishing. By analogy with Minkowski quantization, they are interpreted as annihilation and creation operators of a quantum for the Rindler observer. The corresponding vacuum is defined by $b_{\Omega}^{(\sigma)}\left|0_{\mathrm{R}}\right\rangle=0$, $\forall \sigma, \Omega$.

The question now arises as to how the quantizations (4) (or, equivalently, (7)) and (15) are intertwined. This can be addressed by equating the two field expansions on a spacelike hypersurface lying in the Rindler manifold $R_{ \pm}$and multiplying by $u_{\Omega}^{(\sigma)}$. The following Bogoliubov transformation is then obtained [64]

$b_{\Omega}^{(\sigma)}=\left[1+\mathcal{N}_{\mathrm{BE}}(\Omega)\right]^{1 / 2} d_{\Omega}^{(\sigma)}+\left[\mathcal{N}_{\mathrm{BE}}(\Omega)\right]^{1 / 2} d_{\Omega}^{(-\sigma) \dagger}$, where the Bogoliubov coefficient $\mathcal{N}_{\mathrm{BE}}(\Omega)$ is nothing but the Bose-Einstein distribution function, i.e.

$\mathcal{N}_{\mathrm{BE}}(\Omega)=\frac{1}{e^{2 \pi \Omega}-1}$.

The appearance of a Bogoliubov transformation lies at the heart of the unitary inequivalence between the Fock spaces for the Minkowski and Rindler observers. An immediate consequence of Eq. (17) is that the vacua $\left|0_{R}\right\rangle$ and $\left|0_{M}\right\rangle$ are different from each other, the latter becoming a thermal bath of Rindler particles distributed according to the Bose-Einstein statistics (18). Indeed, a direct calculation of the expectation value of $b_{\Omega}^{(\sigma) \dagger} b_{\Omega^{\prime}}^{\left(\sigma^{\prime}\right)}$ on the vacuum $\left|0_{\mathrm{M}}\right\rangle$ gives

$\left\langle 0_{\mathrm{M}}\left|b_{\Omega}^{(\sigma) \dagger} b_{\Omega^{\prime}}^{\left(\sigma^{\prime}\right)}\right| 0_{\mathrm{M}}\right\rangle=\mathcal{N}(\Omega)_{\mathrm{BE}} \delta_{\sigma \sigma^{\prime}} \delta\left(\Omega-\Omega^{\prime}\right)$.

We point out that the above expression diverges for $\Omega=\Omega^{\prime}$, the reason being that the creation operators $a_{k}^{\dagger}, b_{\Omega}^{\dagger}$ do not produce normalizable states when applied on the respective vacua. To remove such divergency, an approach based on wavepackets (rather than planewaves) should be performed. This has been done explicitly in [64] by using an á la Hawking definition of wavepackets and obtaining a regularized distribution for the Minkowski vacuum condensate.

Now, from Eq. (18) along with the observation that the Rindler energy with respect to the proper time $\tau$ is

$E=a \Omega$,

we infer that the temperature of the vacuum condensate detected by the Rindler observer depends linearly on his acceleration. In fact, we can equivalently rewrite $\mathcal{N}_{\mathrm{BE}}(\Omega)$ in the more familiar form

$\mathcal{N}_{\mathrm{BE}}(\Omega)=\frac{1}{e^{E / T_{\mathrm{U}}}-1}$,

from which we can promptly read the well-known Unruh temperature $T_{\mathrm{U}}=a /(2 \pi)\left(T_{\mathrm{U}}=\hbar a /\left(2 \pi c k_{\mathrm{B}}\right)\right.$ in proper units) [52]. Moreover, a scrupulous analysis of the structure of Minkowski vacuum shows that it appears as a coherent state of Bogoliubov-Bardeen-Cooper-Schrieffer type, in which Rindler particles are pairwise correlated. Specifically, a particle in the positive Rindler wedge $R_{+}$is correlated with an antiparticle in the negative sector $R_{-}$with opposite spatial momentum, and vice-versa. Since the two regions are causally separated from each other, the correlation occurs between space-like separated particles (EPR-like correlation). This feature has been discussed more in-depth in [66].

\subsection{Field quantization in the GUP framework}

The result (21) has been obtained within the framework of the canonical quantum field theory. A heuristic derivation of Unruh effect in quantum mechanics has been proposed in [67, 
68] on the basis of the HUP and thermodynamic arguments. Recently, the same computation has been carried out in the context of the generalized uncertainty principle (1), showing good agreement between the quantum mechanical and field theoretical results [59].

One of the major concerns in dealing with the GUP in quantum field theory is how to implement the condition of minimal length at level of fields and if that even does make physical sense. A first step toward a field theoretical treatment of the GUP has been taken in [59] by looking at the modified structure of the commutator between the ladder operators for the one-dimensional harmonic oscillator. The idea is to exploit the relation between the commutators $\left[a, a^{\dagger}\right]$ and $[x, p]$,

$\left[a, a^{\dagger}\right]=\frac{1}{i}[x, p]$

and derive the deformed commutator on the left side consistently with the GUP (2) for the right side. Following [59], we are led to

$$
\left[a, a^{\dagger}\right]=\frac{1}{1-\alpha}\left[1-\alpha\left(a^{\dagger} a^{\dagger}+a a-2 a^{\dagger} a\right)\right],
$$

where $\alpha$ is related to the GUP deformation parameter $\beta$ by $\alpha=\beta m \omega /\left(2 m_{p}^{2}\right)$, being $\omega$ the oscillation frequency. We notice that the deformation is such that the new commutator is still hermitian, as it should be.

The above considerations can be extended effectively to the quantum field theory. By looking at the free scalar field in the planewave representation as an infinite set of decoupled harmonic oscillators, we can formulate the GUP-version of Eq. (6) as [59]

$$
\begin{aligned}
& {\left[a_{k}, a_{k^{\prime}}^{\dagger}\right]=\frac{1}{1-\tilde{\alpha}}} \\
& \quad \times\left[1-\tilde{\alpha}\left(a_{k}^{\dagger} a_{k^{\prime}}^{\dagger}+a_{k} a_{k^{\prime}}-2 a_{k}^{\dagger} a_{k^{\prime}}\right)\right] \delta\left(k-k^{\prime}\right),
\end{aligned}
$$

where $\tilde{\alpha}=\beta \omega_{k}^{2} /\left(2 m_{p}^{2}\right)=2 \beta \ell_{p}^{2} \omega_{k}^{2}$. The Dirac delta over momenta guarantees that different field modes are still independent from each other. We stress that such a minimalistic assumption makes sense as far as one considers a free field, as it is in our case. More generally, for interacting fields one may consider more elaborate GUP models involving some kind of extra coupling between different field modes. However, this goes beyond the scope of the present analysis.

Now, a similar GUP-based formalism can be built in the boost-mode expansion. Starting from Eq. (11) and imple- menting the GUP corrections as above, we get ${ }^{2}$

$$
\begin{aligned}
& {\left[d_{\Omega}^{(\sigma)}, d_{\Omega^{\prime}}^{\left(\sigma^{\prime}\right) \dagger}\right]} \\
& =\frac{1}{1-\gamma}\left[1-\gamma\left(d_{\Omega}^{(\sigma) \dagger} d_{\Omega^{\prime}}^{\left(-\sigma^{\prime}\right) \dagger}+d_{\Omega}^{(\sigma)} d_{\Omega^{\prime}}^{\left(-\sigma^{\prime}\right)}\right.\right. \\
& \left.\left.\quad-d_{\Omega}^{(\sigma) \dagger} d_{\Omega^{\prime}}^{\left(\sigma^{\prime}\right)}-d_{\Omega}^{(-\sigma) \dagger} d_{\Omega^{\prime}}^{\left(-\sigma^{\prime}\right)}\right)\right] \delta_{\sigma \sigma^{\prime}} \delta\left(\Omega-\Omega^{\prime}\right),
\end{aligned}
$$

with $\gamma=\beta a^{2} \Omega^{2} /\left(2 m_{p}^{2}\right)$. For later convenience, here we rewrite this parameter as $\gamma=\beta \hbar^{2} \omega_{R}^{2} /\left(2 m_{p}^{2} c^{4}\right)$, where $\omega_{R} \equiv a \Omega / c$ (we have restored proper units). More details on the construction of the commutator (25) are given in [59]. Here we just remark that, in passing from Eqs. (24) to (25), the definition of the deformation parameter has been suitably rearranged by replacing the planewave frequency $\omega_{k}$ in $\tilde{\alpha}$ with the boost-mode frequency $a \Omega$ in $\gamma$. Furthermore, the r.h.s. of Eq. (25) has been symmetrized with respect to $\sigma \rightarrow-\sigma$, consistently with the reciprocal symmetry of the two Rindler wedges $R_{ \pm}$. To keep our formalism consistent, henceforth we consider accelerations such that $\gamma$ is at most of order unity, namely, since $\beta \sim 1$, we suppose that $\hbar \omega_{R}$ is at most of order $m_{p} c^{2}$.

If we now recast the Bogoliubov transformation (17) in terms of the new set of $d$-operators, we can assess the effects induced by our GUP model on the Unruh vacuum distribution. From Eq. (25), it follows that [59]

$$
\begin{aligned}
\mathcal{N}_{\beta}(\Omega) & \equiv\left\langle 0_{\mathrm{M}}\left|b_{\Omega}^{(\sigma) \dagger} b_{\Omega^{\prime}}^{\left(\sigma^{\prime}\right)}\right| 0_{\mathrm{M}}\right\rangle \\
& =\frac{1}{\left(e^{2 \pi \Omega}-1\right)(1-\gamma)} \delta_{\sigma \sigma^{\prime}} \delta\left(\Omega-\Omega^{\prime}\right),
\end{aligned}
$$

which shows that the Bose-Einstein statistics is spoilt by a factor dependent upon the GUP parameter $\gamma$. For $\gamma \ll 1$, this becomes

$$
\begin{aligned}
\mathcal{N}_{\beta}(\Omega) & \simeq \frac{1+\gamma}{e^{2 \pi \Omega}-1} \delta_{\sigma \sigma^{\prime}} \delta\left(\Omega-\Omega^{\prime}\right) \\
& =\mathcal{N}_{\mathrm{BE}}(\Omega)(1+\gamma) \delta_{\sigma \sigma^{\prime}} \delta\left(\Omega-\Omega^{\prime}\right) .
\end{aligned}
$$

Clearly, the standard Bose-Einstein distribution is reproduced for $\gamma \rightarrow 0$, consistently with the recovery of the canonical commutator in that limit. Again, the divergency for $\Omega=\Omega^{\prime}$ can be cured by relying on a proper wavepacket approach (see the discussion below Eq. (19)).

In [59] the above result has been interpreted as a signature of the potential thermality breakdown of Unruh effect induced by GUP. Nevertheless, for frequencies $\omega_{R}$ small enough, it has also been shown that the modified vacuum

\footnotetext{
2 It should be noted that models with a minimal length break Lorentz covariance, the reason being that a measured length is not a Lorentz invariant quantity. We then expect that the equivalence between the planewave and boost-mode expansions may not be guaranteed in the presence of the GUP. However, for very tiny deviations from the canonical commutator (i.e. for $\beta p^{2} \ll m_{p}^{2}$ ), we can conjecture for the $d$ operators in Eq. (7) a deformed commutator similar to Eq. (24).
} 
distribution (26) can be rearranged so as to recover a BoseEinstein like profile, provided that the Unruh temperature is assumed to be $\gamma$-dependent. Therefore, in this effective picture, GUP effects on Unruh's prediction would translated into a generalization of the temperature $T_{\mathrm{U}}$, with the expected number spectrum of particles left unchanged.

Our next analysis is based on the deformed spectrum (27). By working in the framework of generalized non-Gaussian statistics, we recast the derivation of the modified Unruh effect in the language of nonextensive Tsallis theory. This allows us to provide an alternative interpretation for the distribution (27) in terms of a departure from extensivity of the statistics of Unruh condensate in the presence of GUP.

\section{3 q-Generalized Tsallis statistics in Unruh effect}

In quantum statistical mechanics it is well-known that the Bose-Einstein distribution describes the way in which a collection of non-interacting, indistinguishable particles occupy a set of available discrete energy states at thermodynamic equilibrium. Specifically, it applies to bosonic particles, that is, particles which do not obey the Pauli exclusion principle. Along with its counterpart for fermions - the FermiDirac statistics - the Bose-Einstein distribution is obtained by maximizing the Boltzmann-Gibbs entropy subject to the constraints that the expected number of particles and the total energy of the system are prescribed.

The usefulness of Boltzmann-Gibbs statistics is founded on the hypothesis that the constituent parts of the system are independent or very weakly (locally) correlated. For such systems, the additive Boltzmann-Gibbs entropy $S=$ $-\sum_{i=1}^{W} p_{i} \log p_{i}$ is to be identified as its (extensive) thermodynamic entropic. Here, the sum runs over the microstates of the system, each having probability $p_{i}$. Of course, the condition $\sum_{i=1}^{W} p_{i}=1$ must be satisfied. On the other hand, systems exhibiting long-range interactions and/or spacetime entanglement, either on quantum or classical grounds, have been proven to lie outside the validity of the BoltzmannGibbs theory, thus prompting the development of a generalized framework that could accommodate their description. In this view it must be seen the formulation of the nonextensive $q$-generalized statistics based on the nonadditive Tsallis entropy [46-49]

$S_{q}=\frac{1-\sum_{i=1}^{W} p_{i}^{q}}{q-1}=\sum_{i=1}^{W} p_{i} \log _{q} \frac{1}{p_{i}}$,

where

$$
\log _{q} z \equiv \frac{z^{1-q}-1}{1-q}, \quad\left(\log _{1} z=\log z\right)
$$

For $q \rightarrow 1$ it is easy to see that $S_{q}$ reduces to BoltzmannGibbs entropy. Furthermore, for probabilistically independent systems such that $p_{i j}^{A+B}=p_{i}^{A} p_{j}^{B}, \forall(i, j)$, the definition (28) leads to

$S_{q}(A+B)=S_{q}(A)+S_{q}(B)+(1-q) S_{q}(A) S_{q}(B)(30)$

which indicates the superadditive or subadditive regime of Tsallis entropy, depending on whether $q<1$ or $q>1$. The parameter $q \in \mathbb{R}^{+}$represents the degree of nonextensivity of Tsallis entropy. In particular, the larger the deviation of $q$ from unity, the higher the degree of nonextensivity, and vice-versa. Beyond formal aspects, it is worth noting that Tsallis statistics has provided encouraging results in describing a broad class of complex systems, such as self-gravitating stellar systems [69], black holes [48], the cosmic background radiation [70], low-dimensional dissipative systems [49] and polymer chains [71], among others. Recently, an effective description of field mixing based on Tsallis entropy has been proposed in [72].

The $q$-generalized entropy (28) naturally leads to nontrivial modifications of the standard predictions of BoltzmannGibbs theory. For instance, in [73] it has been discussed to what extent the nonextensivity affects the core thermodynamic relations, including the zero-th law of thermodynamics and the concepts of temperature and pressure. Similarly, in [54-58] Tsallis entropy has been used to derive the following generalized Bose-Einstein distribution

$\mathcal{N}_{q}(E)=\frac{1}{[1+(q-1) \beta E]^{1 /(q-1)}-1}$,

which is obtained via the maximum entropy principle applied to Eq. (28). Here $\beta=1 / T$, with $T$ being related (but not exactly equal) to the effective temperature of the system. More details on this will be discussed below. Again, the $q \rightarrow$ 1 limit gives back the Bose-Einstein distribution (18).

To make sense as a distribution function, $\mathcal{N}_{q}(E)$ must be non-negative and real-valued. This gives rise to the following constraints

$\begin{cases}0 \leq E \leq[(1-q) \beta]^{-1} & \text { for } q<1 \\ E \geq 0 & \text { for } q>1 .\end{cases}$

At this stage, it should be emphasized that Eq. (31) is only an approximation [74]. Indeed, for arbitrary values of $q$ an analytic expression for the exact generalized distribution cannot be derived. However, the use of Eq. (31) is well-justified for systems with a large total number of particles (such as fields) and at very low temperatures (see [74] for more details). Thus, since the Unruh temperature $T_{\mathrm{U}}$ takes extremely small values even for large accelerations (notice that an acceleration $a \sim 10^{20} \mathrm{~m} / \mathrm{s}^{2}$ is barely enough to reach a temperature $T_{\mathrm{U}} \sim 1 \mathrm{~K}$ ), we can safely assume Eq. (31) as a starting point for revisiting the field theoretical derivation of the GUP-modified Unruh effect in Tsallis statistics. 
In this context, we remark that a first effort to establish a connection between the Unruh effect in the GUP framework and non-Gaussian statistics has been made in [51]. There, the strategy was to explore how the prescription (1) enters into the phase space structure and modifies the elementary cell volume. On this basis, it was shown that $q$-generalized statistics with a quadratic correction over Gaussian profile arise quite naturally from the GUP (1), resulting in a nontrivial $q$ dependent modification of the energy/temperature relation for a free particle (generalized equipartition theorem). By identifying the modified Unruh temperature obtained in this way with the corresponding quantum mechanical expression derived heuristically from the GUP in [59], the following link was exhibited

$q=1+\frac{54 \beta}{17 \pi^{2}} \ell_{p}^{2} a^{2}=1+\frac{27 \beta}{34 \pi^{2}} \frac{\hbar^{2} a^{2}}{m_{p}^{2} c^{6}}$,

where in the last step we have expressed the Planck length in terms of the Planck mass and restored proper units. As expected, the departure $|q-1|$ from the Gaussian behavior is proportional to the GUP parameter $\beta$, so that $q \rightarrow 1$ for $\beta \rightarrow 0$ (or, equivalently, for accelerations such that $\hbar a \ll$ $\left.m_{p} c^{3}\right)$.

The above relation relies on the comparison between the modified Unruh temperature arising in nonextensive statistics and that derived by the GUP. Strictly speaking, it holds true in quantum mechanics, where GUP corrections on Unruh effect can indeed be encoded in a deformation of the temperature. On the other hand, as discussed below Eq. (27), in quantum field theory one can identify a temperature of the vacuum condensate only in a particular energy regime. The question thus arises as to how the correspondence (33) would appear in quantum field theory.

To see this, let us come back to the generalized BoseEinstein distribution (31). Of course, since GUP-induced deviations from the standard spectrum are expected to be very small, it is reasonable to expand Eq. (31) for tiny departures of $q$ from unity. To the leading order, we then obtain

$\mathcal{N}_{q}(E) \simeq \frac{1}{e^{\beta E}-1}+\frac{1}{8}(\beta E)^{2} \operatorname{csch}^{2}\left(\frac{\beta E}{2}\right)(q-1)$.

In order to cast this expression in a form which resembles the modified particle spectrum (27), we resort to the definition (20) of Rindler proper energy and set $\beta=1 / T_{\mathrm{U}}=$ $2 \pi / a$, with $T_{\mathrm{U}}$ being the Unruh temperature. This yields

$\mathcal{N}_{q}(\Omega) \simeq \mathcal{N}_{\mathrm{BE}}(\Omega)+\frac{\pi^{2}}{2} \Omega^{2} \operatorname{csch}^{2}(\pi \Omega)(q-1)$,

where the zeroth-order term $N_{\mathrm{BE}}$ is the Bose-Einstein spectrum (21). In spite of the minimalistic expansion, we notice that the generalized Bose-Einstein distribution based on Tsallis entropy exhibits a nontrivial energy dependence.
To show the correspondence between the GUP and Tsallis statistics, let us now compare Eqs. (27) and (35). A straightforward calculation gives (to the leading order)

$$
\begin{aligned}
q & =1+\frac{\beta}{4 \pi^{2}} \frac{a^{2}}{m_{p}^{2}}\left(1-e^{-2 \pi \Omega}\right), \\
& =1+\frac{\beta}{4 \pi^{2}} \frac{\hbar^{2} a^{2}}{m_{p}^{2} c^{6}}\left(1-e^{-\hbar \omega_{R} / k_{\mathrm{B}} T_{\mathrm{U}}}\right),
\end{aligned}
$$

where in the last step we have restored proper units.

Some comments are needed here: first, we note that the entropic index $q$ exhibits a running behavior as a function of the field-mode frequencies (i.e. the energy scale). This behavior should not be entirely surprising, as it is known to arise quite naturally for quantum field theoretical or quantum gravity systems, where the renormalization group is applied. In those cases the coupling constants (including cosmological and gravitational coupling constants) are running with the energy scale. Here, the $q$-index appearing in the description of the generalized entropy of the system is expected to be running, since entropy is a measure of the physical degrees of freedom of the systems, which indeed turn out to be scale-dependent in QFT. Thus, the above outcome sinks its physical roots in quantum field theory considerations, which although not taken into account by Tsallis in his original formulation of nonextensive thermodynamics, are in principle mandatory when one tries to extend Tsallis entropy to a more general QFT framework. The same scenario with a varying nonextensive parameter has been recently addressed in [75] in the context of modified cosmological models. The main difference is that, in that case, the authors assume $a b$ initio a running behavior for Tsallis parameter, while here we have found such a behavior as a result of the requirement of consistency with the GUP framework. Along this line, a similar achievement has been displayed in [72] in connection with the description of the superposition of fields with different masses (field mixing). We also point out that, for fixed $\Omega, q$ increases monotonically with $\beta$. This implies a one-to-one correspondence between the GUP parameter and a linear function of it.

For small Rindler energies $\hbar \omega_{R}$ with respect to $k_{\mathrm{B}} T_{\mathrm{U}}$, i.e. $\hbar \omega_{R} \ll k_{\mathrm{B}} T_{\mathrm{U}} \Longrightarrow \Omega \ll 1 /(2 \pi)$, Eq. (36) can be approximated as

$q=1+\frac{\beta}{2 \pi} \frac{\hbar^{2} a^{2} \Omega}{m_{p}^{2} c^{6}}$,

which resembles Eq. (33), except for the $\Omega$-dependence of $q-1$. This could be somehow expected, since the correspondence (37) has been derived by considering the modified vacuum distribution involving different-frequency field modes, while Eq. (33) relies on quantum mechanical considerations, i.e. it should be regarded as being valid for a single mode with a well-defined frequency. Notice that the exact match would 
be got for $\Omega \simeq 1 / 2$, which however lies outside the regime in which Eq. (37) holds true. Since $\Omega>0$, from Eq. (36) or Eq. (37) we also infer that $q>1$, which is indicative of the subadditive feature of Tsallis entropy for the modified vacuum distribution (see Eq. (30)). In turn, this ensures that no special restriction on Rindler frequency arises from Tsallis's prescription, according to Eq. (32). As an aside, it should be mentioned that the opposite scenario would occur for negative $\beta$. In that case, the entropic index $q$ would be less than unity, thereby leading to a superadditive regime of Tsallis entropy and a frequency cutoff beyond which our description cannot be applied. Nevertheless, we stress that the GUP (1) with negative $\beta$ does not entail any minimal length (see the discussion below Eq. (1)). Since, in our framework, deviations from Gaussian statistics are ascribed to the emergence of a fundamental scale and to the ensuing change of the elementary phase-space cell volume, a GUP model with $\beta<0$ would be unjustified for our purposes. Clearly, for $\Omega \rightarrow 0$ the Bose-Einstein spectrum with $q=1$ is recovered. Thus, within the above framework, the zerofrequency Rindler modes are well-described by the standard Boltzmann-Gibbs thermostatistics, even in the presence of the GUP.

On the other hand, for $\hbar \omega_{R} \simeq k_{\mathrm{B}} T_{\mathrm{U}} \Longrightarrow \Omega \simeq 1 /(2 \pi)$, which is the frequency at which the vast majority of Unruh quanta are expected to be emitted [59], Eq. (36) gives

$q=1+\frac{\beta}{4 \pi^{2}} \frac{\hbar^{2} a^{2}}{m_{p}^{2} c^{6}}\left(1-e^{-1}\right) \simeq 1+0.02 \beta \frac{\hbar^{2} a^{2}}{m_{p}^{2} c^{6}}$,

which agrees with Eq. (33), up to a numerical factor. Last, for Rindler frequencies such that $k_{\mathrm{B}} T_{\mathrm{U}} \ll \hbar \omega_{R} \ll m_{p} c^{2} \Longrightarrow$ $1 /(2 \pi) \ll \Omega \ll m_{p} c^{3} /(\hbar a)$, the term in the brackets in Eq. (36) reduces to unity. ${ }^{3}$ In this regime, we obtain the frequency-independent relation

$q=1+\frac{\beta}{4} \frac{\hbar^{2} a^{2}}{\pi^{2} m_{p}^{2} c^{6}}$,

which is analogous to Eq. (33), the only difference again being a simple numerical factor. Therefore, in light of these considerations, we can state that the quantum mechanical relation (33) provides the high-energy approximation of the more general field theoretical correspondence (36).

From Eq. (39), we can also estimate an upper bound for the deviation $q-1$. Toward this end, we observe that the departure of $q$ from unity grows quadratically with the acceleration. In this context, we consider an accelerated particlelike detector coupled to the scalar field. We refer to [76,77],

\footnotetext{
${ }^{3}$ We recall that the upper bound on the Rindler frequency $\Omega$ must be required in order to fall within the domain of validity of the approximation (27), which underlies the derivation of Eq. (36). To give some numerical estimation, we point out that such bound is still of order $m_{p} c^{3} /(\hbar a) \simeq 10^{31}$ for $a \sim 10^{20} \mathrm{~m} / \mathrm{s}^{2}$.
}

where it was derived an upper limit to the acceleration of any massive elementary particle by combining basic notions of special relativity and quantum theory. ${ }^{4}$ As a result, it was shown that the maximal value $a_{\max }$ would be reached for a particle of Planck mass and takes the value $a_{\max }=m_{p} c^{3} / \hbar$. Then, by plugging $a=a_{\max }$ in Eq. (39), we obtain

$q \lesssim \frac{11}{9} \Longrightarrow q-1 \lesssim \frac{2}{9} \approx 0.22$,

where we have used for $\beta$ the theoretical bound derived in [21] in the context of Unruh effect in Caianiello's theory of maximal acceleration, i.e. $\beta=8 \pi^{2} / 9$. It is interesting to note that the same value for the Tsallis index has been recently obtained by cosmic ray observations $(q=11 / 9$ [78]). Similar estimations have also been inferred by the quark coalescence fit to Relativistic Heavy Ion Collider $(q \approx 1.2)$ [79] and from semiclassical considerations of black holes in quantum geometry $(q \approx 1.218[80])$.

As a final remark, we emphasize that in the above analysis the quantity $T_{\mathrm{U}}$ appearing in Eq. (36) should no longer be regarded as the temperature of the modified vacuum distribution. Indeed, as shown in [73], in contrast with the extensive case, the physical temperature is not $\left(k_{\mathrm{T}} \beta\right)^{-1}$, but

$T_{p h y s}=\frac{1}{k_{\mathrm{T}} \beta^{*}}=\left(1+\frac{1-q}{k_{\mathrm{T}}} S_{q}\right) \frac{1}{k_{\mathrm{T}} \beta}$,

where $k_{\mathrm{T}}$ is a constant which may depend on $q$ and becomes the Boltzmann constant $k_{\mathrm{B}}$ in the extensive limit $q \rightarrow 1$. More generally, there is room for considering a dependence on the size system as well.

To enhance the connection between the GUP and Tsallis framework, in the Appendix we extend similar considerations to black hole thermodynamics.

\section{Conclusions and outlook}

It is a well-established fact that a vast class of physical phenomena can be adequately described by distributions deviating from Gaussian statistics. To date, it is understood that these statistics may originate from different processes, such as fluctuations [81], stochastic network approach [82] or a modification of the phase space cell volume [41,42]. Among the various examples of non-Gaussian statistics, the $q$-generalized Tsallis statistics has proved to be a useful tool for studying complex systems characterized by long-range interactions and/or long-range microscopic memories. More

\footnotetext{
${ }^{4}$ Strictly speaking, the derivation of Caianiello's maximal acceleration relies on the use of an energy/velocity version of the standard HUP [76, 77]. Thus, one would expect that also $a_{\max }$ may acquire a $\beta$-dependent correction in the context of GUP. However, from Eq. (39) it is clear that such correction would lead to a quadratic term in $\beta$, which can be neglected in our first-order approximation.
} 
recent, but already numerous, are the applications in high energy physics [48,49,69-72,75].

Inspired by the result of [51], in this work we have substantiated the idea that the emergence of a minimal length induced by the GUP introduces a change in the volume of the elementary phase space cell, which can be naturally translated into a departure of the statistics from a pure Gaussian behavior. As a specific playground, we have considered the Unruh effect, which has been recently studied in the context of quantum field theory with the GUP [59]. In this framework, we have shown that the GUP-modified distribution of Unruh condensate can be mapped into the $q$-generalized Bose-Einstein distribution based on Tsallis entropy. In turn, this allows establishing a link between the $q$-entropic index and the GUP deformation parameter $\beta$, which generalizes to quantum field theory the correspondence previously found in quantum mechanics in [51]. Clearly, the result obtained in Eq. (36) is a first approximation. Indeed, a more rigorous treatment should be based on the employment of a Lorentz covariant GUP, which reduces to its familiar non-relativistic version at low energies and incorporates a minimum length. Furthermore, one should perform exact calculations, which go beyond the assumptions of small deformation of the commutator and/or small deviation from extensivity. Work along these directions is presently under active investigation.

Preliminary arguments supporting the connection between the GUP and Tsallis statistics have also been provided within the framework of black hole thermodynamics. In this case, we have compared the GUP modified black hole entropy and the nonextensive entropy derived from Tsallis's prescription, leading again to a nontrivial monotonic relation between $q$ and $\beta$.

In passing, we notice that, in recent years, Tsallis entropy has been largely used in the cosmological framework [83,84]. In particular, in [83] a generalization of the standard holographic dark energy has been formulated based on Tsallis nonextensive entropy, while in [84] modified Friedmann equations have been derived through nonextensive horizon thermodynamics. A similar analysis has been carried out in [85] in the context of a deformed uncertainty relation with UV cutoffs on both the length and momentum, showing that the induced extra terms in the cosmological equations naturally lead to the realization and stability of the Einstein static universe, and provide a mechanism to a phase transition to the inflationary era and the subsequent thermal history of the universe. In the light of the established correspondence between the GUP and Tsallis statistics, it would be interesting to see whether a similar connection can also be recovered in this cosmological scenario. One possibility might be to compare the solutions of the modified cosmological equations arising in the two frameworks. By requiring consistency, one can then infer the relation between the deformation parameter of the uncertainty relation and Tsallis index. More general, however, we expect that deviations from the HUP at cosmic scale become relevant in the context of the Extended Uncertainty Principle (EUP) [86-88], applications of which have been recently addressed in [89-91]. This aspect will be analyzed more in-depth elsewhere.

Finally, let us observe that, since the GUP provides us with an effective model to tackle gravity at Planck scale, our result is to be understood in the broader context of a possible description of Planck-scale gravitational physics based on Tsallis theory. This is indeed in line with the achievement of [92], where the Schrödinger-type minimum-uncertainty GUP coherent states have been derived both in the momentum and position representation, showing that in the latter case they coincide with Tsallis probability amplitudes. Furthermore, for the $\beta<0$ scenario, the GUP (1) has also been reformulated in terms of one-parameter class of Tsallis entropy-power based uncertainty relations, which are saturated by the GUP coherent states. Since coherent states are privileged states in the transition from quantum to classical reality (as they are the only states that remain pure after decoherence processes), the established correspondence suggests that gravity in the semiclassical quantum regime can be naturally described by nonextensive Tsallis thermostatistics through its connection with the GUP. The investigation of this point clearly deserves more attention and will be deepened in a future work.

Acknowledgements The author is grateful to Costantino Tsallis (Centro Brasileiro de Pesquisas Fisicas, Brazil) for helpful conversations. The author would also like to thank the anonymous Referees for their insights and suggestions, which helped to improve the quality of the manuscript.

Data Availability Statement This manuscript has no associated data or the data will not be deposited. [Authors' comment: Data sharing not applicable to this article as no datasets were generated or analyzed during the current study.]

Open Access This article is licensed under a Creative Commons Attribution 4.0 International License, which permits use, sharing, adaptation, distribution and reproduction in any medium or format, as long as you give appropriate credit to the original author(s) and the source, provide a link to the Creative Commons licence, and indicate if changes were made. The images or other third party material in this article are included in the article's Creative Commons licence, unless indicated otherwise in a credit line to the material. If material is not included in the article's Creative Commons licence and your intended use is not permitted by statutory regulation or exceeds the permitted use, you will need to obtain permission directly from the copyright holder. To view a copy of this licence, visit http://creativecomm ons.org/licenses/by/4.0/.

Funded by SCOAP ${ }^{3}$.

\section{Appendix A: Generalized black hole thermodynamics}

To substantiate the connection between deformations of the uncertainty relation and Tsallis statistics, in this Appendix 
we consider GUP-black hole thermodynamics. It should be said that, since a consistent field theoretical treatment of this theory in the context of GUP is missing, in this case we can only provide quantum mechanical arguments. Following the same scheme as in Sect. 3, we first focus on the derivation of some black hole thermodynamic relations based on the GUP. We then move to Tsallis theory. Comparison between the frameworks still enables us to infer a link between the GUP deformation parameter and Tsallis nonextensive index.

Let us start with the derivation of the GUP-modified black hole temperature as done in [93]. For states that saturate the inequality (1), by solving for $\Delta p$ we obtain

$\Delta p=\frac{\Delta x m_{p}^{2}}{\beta}\left(1-\sqrt{1-\beta \frac{1}{\Delta x^{2} m_{p}^{2}}}\right)$,

where we have considered the only solution that recovers the standard quantum mechanics in the limit of $\beta \rightarrow 0$. To the leading order, this becomes

$\Delta p \simeq \frac{1}{2 \Delta x}+\frac{\beta}{8} \frac{1}{\Delta x^{3} m_{p}^{2}}$.

The computation of the modified Hawking temperature proceeds as follows. Consider a spherically symmetric black hole of mass $M$ and Schwarzschild radius $r_{s}=2 M G$, with $G$ being the Newton's gravitational constant. Heuristically, we can depict black hole evaporation as the result of the conversion of vacuum fluctuations around the black hole surface into pairs of particles (mostly photons), the one being trapped inside the horizon, the other escaping in the form of Hawking radiation at the expense of the source mass. The characteristic energy of these particles can be estimated by using the uncertainty principle and setting the position uncertainty to be of order of Schwarzschild radius, i.e. $\Delta x=\mu r_{s}$, where the numerical factor $\mu$ will be fixed a posteriori [93]. From Eq. (A2), we obtain

$\Delta E \simeq \frac{1}{4 \mu M G}+\frac{\beta}{64 \mu^{3}} \frac{1}{M^{3} G^{2}}$.

If we interpret this energy fluctuation as a classical thermal agitation, from the equipartition theorem we derive the temperature of the emitted radiation as

$\Delta E \simeq \frac{3}{2} T_{\mathrm{GUP}} \Longrightarrow T_{\mathrm{GUP}} \simeq \frac{1}{6 \mu M G}+\frac{\beta}{96 \mu^{3}} \frac{1}{M^{3} G^{2}}(\mathrm{~A} 4)$

At this stage, we notice that $\mu$ can be fixed by requiring consistency with the Hawking temperature $T_{\mathrm{H}}=1 /(8 \pi M G)$ in the HUP limit, which yields $\mu=4 \pi / 3$. By plugging into the above expression, we are finally led to

$T_{\mathrm{GUP}} \simeq T_{\mathrm{H}}\left(1+\frac{9 \beta}{256 \pi^{2}} \frac{1}{M^{2} G}\right)=T_{\mathrm{H}}\left(1+\frac{9 \beta}{256 \pi^{2}} \frac{m_{p}^{2}}{M^{2}}\right)$, which provides us with the GUP-modified expression of Hawking temperature. In passing, we mention that, by contrast to the standard quantum mechanics, where the Hawking temperature blows up as the black hole completely evaporates away, the GUP with $\beta>0$ predicts that black hole stop shrinking at around $M \sim m_{p}$, leaving behind an inert remnant with finite temperature [93]. On the other hand, for $\beta<0$ black holes still exhibit a finite temperature in their final stage, despite not having any lower bound on the size [94].

We now turn to the computation of the black hole entropy. According to the second law of thermodynamics, it can be derived by integrating $d S=d M / T$. Clearly, for $T=$ $T_{\mathrm{H}}$ one obtains the Bekenstein-Hawking formula $S_{\mathrm{BH}}=$ $A_{\mathrm{H}} /\left(4 \ell_{p}^{2}\right)=4 \pi M^{2} / m_{p}^{2}$, where $A_{\mathrm{H}}=4 \pi r_{s}^{2}$ is the black hole horizon area. Conversely, the modified entropy that follows from the temperature (A5) is given to the leading order in $\beta$ by

$$
\begin{aligned}
S_{\mathrm{GUP}} & \simeq S_{\mathrm{BH}}+\frac{9 \beta}{32 \pi} \log \left(\frac{m_{p}}{M}\right)+\text { const } \\
& =S_{\mathrm{BH}}-\frac{9 \beta}{64 \pi} \log \left(S_{\mathrm{BH}}\right)+\text { const }^{\prime},
\end{aligned}
$$

where the constant can be fixed by normalizing the entropy to zero at $M=m_{p}$ [93].

Before going further, it is worth noting that the same logarithmic correction to black hole entropy arises from oneloop effects of quantum matter fields near a black hole. This has been shown explicitly for the case of $N_{s}$ massless fields of spin $s$ close to a Schwarzschild black hole [95] and for a single scalar field near an extreme Reissner-Nordström source [96]. Recently, great attention has been devoted to non-perturbative calculations of purely quantum gravity corrections using both loop quantum gravity [97] and string theory [98] (see [99] for a comprehensive review on the topic). Remarkably, in all these cases the modified black hole entropy can be cast in the form

$S=S_{\mathrm{BH}}+\alpha \log \left(S_{\mathrm{BH}}\right)+\kappa$,

with $\alpha$ and $\kappa$ yet to be determined. In particular, there is quite a lively debate on how to fix the logarithmic pre-factor $\alpha$ (e.g. with $\kappa=0$ ), as it appears to be strongly modeldependent. However, several approaches predict that $\alpha<0$ $[97,100]$. In this regard, we can link such coefficient to the GUP parameter $\beta$ by comparing Eqs. (A6) and (A7). This yields $\alpha=-9 \beta /(64 \pi)$, which implies that consistency between the two models is obtained, provided that $\beta>0$. In this regard, we emphasize that a similar comparative analysis can also be carried out with reference to the recently introduced Barrow formulation of black hole entropy $S_{\mathrm{B}}=\left(\frac{A}{4 \ell_{p}^{2}}\right)^{1+\Delta / 2}$ [101], where the Barrow exponent $0 \leq \Delta \leq 1$ quantifies the effects of quantum fluctua- 
tions of spacetime geometry on black hole thermodynamics (in particular, $\Delta=0$ represents the usual classical spacetime, while $\Delta=1$ features the maximal fractal nature of spacetime induced by fluctuations). By expanding for small values of $\Delta$ and comparing with Eq. (A6), one can in principle derive the correspondence between $\beta$ and $\Delta$, obtaining a proportional relationship between the two parameters. We also remark that Barrow black hole corrected-entropy model has been studied in connection with Tsallis nonextensivity in [102].

Let us now compare Eq. (A6) with the modified black hole entropy based on Tsallis statistics. From [48], the latter reads

$S_{q}=\ln _{q} W\left(\frac{M}{m_{p}}\right)$,

where the total number of internal configurations $W$ is such that $\log W=S_{\mathrm{BH}}$, in order to recover the standard Bekenstein-Hawking formula in the extensive limit.

Following the same considerations as in Sect. 3, we expand the above formula for small deviations from extensivity. After some algebra, we obtain

$$
\begin{aligned}
S_{q} & =\frac{W^{1-q}-1}{1-q} \\
& \simeq \log W-\frac{1}{2}(\log W)^{2}(q-1),
\end{aligned}
$$

which can be equivalently rewritten as

$S_{q}=S_{\mathrm{BH}}+\frac{S_{\mathrm{BH}}^{2}}{2}(1-q)$.

By requiring consistency with the GUP-modified expression (A6), we get

$$
\begin{aligned}
q & =1+\frac{9 \beta}{32 \pi} \frac{\log S_{\mathrm{BH}}}{S_{\mathrm{BH}}^{2}} \\
& =1+\frac{9 \beta}{512 \pi^{3}} \frac{m_{p}^{4}}{M^{4}} \log \left(4 \pi \frac{M^{2}}{m_{p}^{2}}\right) .
\end{aligned}
$$

As for the previous case, $q$ is a monotonically increasing function of $\beta$ and exhibits a running behavior as a function of the energy scale (the rôle of the acceleration is now played by the black hole mass). Notice that, for large mass respect to $m_{p}$, we have $q \rightarrow 1$. This is consistent with the fact that, far from the Planck scale, GUP effects become negligible. Since in our picture we are mapping GUP onto Tsallis prescription, it is therefore expected that also the standard BoltzmannGibbs theory with $q=1$ is recovered in this limit. Of course, the same occurs for $\beta \rightarrow 0$.

\section{References}

1. D. Amati, M. Ciafaloni, G. Veneziano, Phys. Lett. B 197, 81 (1987)
2. D.J. Gross, P.F. Mende, Phys. Lett. B 197, 129 (1987)

3. D. Amati, M. Ciafaloni, G. Veneziano, Phys. Lett. B 216, 41 (1989)

4. K. Konishi, G. Paffuti, P. Provero, Phys. Lett. B 234, 276 (1990)

5. M. Maggiore, Phys. Lett. B 319, 83 (1993)

6. S. Capozziello, G. Lambiase, G. Scarpetta, Int. J. Theor. Phys. 39 , $15(2000)$

7. A. Kempf, G. Mangano, R.B. Mann, Phys. Rev. D 52, 1108 (1995)

8. M. Bojowald, A. Kempf, Phys. Rev. D 86, 085017 (2012)

9. F. Scardigli, R. Casadio, Class. Quantum Gravity 20, 3915 (2003)

10. F. Scardigli, Phys. Lett. B 452, 39 (1999)

11. R.J. Adler, D.I. Santiago, Mod. Phys. Lett. A 14, 1371 (1999)

12. R. Casadio, R. Garattini, F. Scardigli, Phys. Lett. B 679, 156 (2009)

13. P. Jizba, H. Kleinert, F. Scardigli, Phys. Rev. D 81, 084030 (2010)

14. R. Casadio, F. Scardigli, Eur. Phys. J. C 74, 2685 (2014)

15. S. Das, E.C. Vagenas, Phys. Rev. Lett. 101, 221301 (2008)

16. F. Brau, J. Phys. A 32, 7691 (1999)

17. P. Pedram, K. Nozari, S.H. Taheri, JHEP 1103, 093 (2011)

18. F. Scardigli, R. Casadio, Eur. Phys. J. C 75, 425 (2015)

19. P. Bosso, S. Das, R.B. Mann, Phys. Rev. D 96, 066008 (2017)

20. F. Scardigli, G. Lambiase, E. Vagenas, Phys. Lett. B 767, 242 (2017)

21. G.G. Luciano, L. Petruzziello, Eur. Phys. J. C 79, 283 (2019)

22. I. Kuntz, R. Da Rocha, Eur. Phys. J. C 80, 478 (2020)

23. L. Buoninfante, G. Lambiase, G.G. Luciano, L. Petruzziello, Eur. Phys. J. C 80, 853 (2020)

24. L. Buoninfante, G.G. Luciano, L. Petruzziello, Eur. Phys. J. C 79, 663 (2019)

25. I. Pikovski, M.R. Vanner, M. Aspelmeyer, M.S. Kim, C. Brukner, Nat. Phys. 8, 393 (2012)

26. A.F. Ali, S. Das, E.C. Vagenas, Phys. Rev. D 84, 044013 (2011)

27. M. Bawaj, C. Biancofiore, M. Bonaldi, F. Bonfigli, A. Borrielli, G. Di Giuseppe, L. Marconi, F. Marino, R. Natali, A. Pontin et al., Nat. Commun. 6, 7503 (2015)

28. P.A. Bushev, J. Bourhill, M. Goryachev, N. Kukharchyk, E. Ivanov, S. Galliou, M.E. Tobar, S. Danilishin, Phys. Rev. D 100, 066020 (2019)

29. F. Marin, F. Marino, M. Bonaldi, M. Cerdonio, L. Conti, P. Falferi, R. Mezzena, A. Ortolan, G.A. Prodi, L. Taffarello et al., Nat. Phys. 9, $71(2013)$

30. P. Bosso, S. Das, R.B. Mann, Phys. Lett. B 785, 498 (2018)

31. G.G. Luciano, L. Petruzziello, Eur. Phys. J. Plus 136, 179 (2021)

32. F. Scardigli, J. Phys. Conf. Ser. 1275, 012004 (2019)

33. L.N. Chang, D. Minic, N. Okaruma, T. Takeuchi, Phys. Rev. D 65, 125028 (2002)

34. D. Mania, M. Maziashvili, Phys. Lett. B 705, 521 (2011)

35. J.C. Niemeyer, Phys. Rev. D 65, 083505 (2002)

36. M. Lubo, Phys. Rev. D 68, 125004 (2003)

37. B. Vakili, M.A. Gorji, J. Stat. Mech. 1210, P10013 (2012)

38. K. Nozari, B. Fazlpour, Gen. Relativ. Gravit. 38, 1661 (2006)

39. T. Fityo, Phys. Lett. A 372, 5872 (2008)

40. M. Abbasiyan-Motlaq, P. Pedram, J. Stat. Mech. 1408, P08002 (2014)

41. S. Abe, Europhys. Lett. 90, 50004 (2010)

42. P. Quarati, M. Lissia, Entropy 15, 4319 (2013)

43. B. Andresen, Europhys. Lett. 92, 40005 (2010)

44. S. Abe, Europhys. Lett. 92, 40006 (2010)

45. F. Quarati, P. Quarati, Entropy 5, 239 (2003)

46. C. Tsallis, J. Stat. Phys. 52, 479 (1988)

47. C. Tsallis, Introduction to Non-Extensive Statistical Mechanics: Approaching a Complex World (Springer, Berlin, 2009)

48. C. Tsallis, L.J.L. Cirto, Eur. Phys. J. C 73, 2487 (2013)

49. M.L. Lyra, C. Tsallis, Phys. Rev. Lett. 80, 53 (1998)

50. J. Havrda, F. Charvát, Kybernetika 3, 1 (1967)

51. H. Shababi, K. Ourabah, Eur. Phys. J. Plus 135, 697 (2020) 
52. W.G. Unruh, Phys. Rev. D 14, 870 (1976)

53. J.H. Jeans, Philos. Trans. A Math. Phys. Eng. Sci. 199, 1 (1902)

54. F. Büyükkiliç, D. Demirhan, Phys. Lett. A 181, 24 (1993)

55. F. Büyükkiliç, D. Demirhan, A. Güleç, Phys. Lett. A 197, 209 (1995)

56. U. Timakli, F. Büyükkiliç, D. Demirhan, Physica A 240, 657 (1997)

57. Q.A. Wang, M. Pezeril, A. Le Mèhautè, Physica A 278, 337 (2000)

58. J. Chen, Z. Zhang, G. Su, L. Chen, Y. Shu, Phys. Lett. A 300, 65 (2002)

59. F. Scardigli, M. Blasone, G. Luciano, R. Casadio, Eur. Phys. J. C 78, 728 (2018)

60. P.M. Alsing, P.W. Milonni, Am. J. Phys. 72, 1524 (2004)

61. B.S. DeWitt, Quantum gravity: the new synthesis, in General Relativity: An Einstein Centenary Survey, ed. by S.W. Hawking, W. Israel (Cambridge University Press, Cambridge, 1979)

62. M. Blasone, G. Lambiase, G.G. Luciano, Phys. Rev. D 96, 025023 (2017)

63. M. Blasone, G. Lambiase, G.G. Luciano, J. Phys. Conf. Ser. 956, 012021 (2018)

64. S. Takagi, Prog. Theor. Phys. Suppl. 88, 1 (1986)

65. N.D. Birrell, P.C.W. Davies, Quantum Fields in Curved Space (Cambridge University Press, Cambridge, 1982)

66. W.G. Unruh, R.M. Wald, Phys. Rev. D 29, 1047 (1984)

67. F. Scardigli, Nuovo Cim. B 110, 1029 (1995)

68. J. Giné, EPL 121, 10001 (2018)

69. A.R. Plastino, A. Plastino, Phys. Lett. A 174, 384 (1993)

70. C. Tsallis, F.C. Sa Barreto, E.D. Loh, Phys. Rev. E 52, 1447 (1995)

71. P. Jizba, J. Korbel, V. Zatloukal, Phys. Rev. E 95, 022103 (2017)

72. G.G. Luciano, M. Blasone, arXiv:2104.00395 [hep-th]

73. S. Abe, S. Martínez, F. Pennini, A. Plastino, Phys. Lett. A 281, $126(2001)$

74. Q.A. Wang, Le Mèhautè, Phys. Lett. A 2, 35, 222 (1997)

75. S. Nojiri, S.D. Odintsov, E.N. Saridakis, Eur. Phys. J. C 79, 242 (2019)
76. E.R. Caianiello, Lett. Nuovo Cim. 32, 65 (1981)

77. E.R. Caianiello, Lett. Nuovo Cim. 41, 370 (1984)

78. C. Beck, Eur. Phys. J. A 40, 267 (2009)

79. T.S. Biro, V.G. Czinner, Phys. Lett. B 726, 861 (2013)

80. K. Mejrhit, R. Hajji, Eur. Phys. J. C 80, 1060 (2020)

81. G. Wilk, Z. Wlodarczyk, Phys. Rev. Lett. 84, 2770 (2000)

82. G. Wilk, Z. Wlodarczyk, Acta Phys. Pol. B 35, 871 (2004)

83. E.N. Saridakis, K. Bamba, R. Myrzakulov, F.K. Anagnostopoulos, JCAP 12, 012 (2018)

84. A. Lymperis, E.N. Saridakis, Eur. Phys. J. C 78, 993 (2018)

85. M. Khodadi, K. Nozari, E.N. Saridakis, Class. Quantum Gravity 35, 015010 (2018)

86. B. Bolen, M. Cavaglia, Gen. Relativ. Gravit. 37, 1255 (2005)

87. Mi Park, Phys. Lett. B 659, 698 (2008)

88. S. Mignemi, Mod. Phys. Lett. A 25, 1697 (2010)

89. T. Zhu, J.R. Ren, M.F. Li, Phys. Lett. B 674, 204 (2009)

90. J. Mureika, Phys. Lett. B 789, 88 (2019)

91. J. Giné, G.G. Luciano, Eur. Phys. J. C 80, 1039 (2020)

92. P. Jizba, G. Lambiase, G.G. Luciano, L. Petruzziello, To appear

93. R.J. Adler, P. Chen, D.I. Santiago, Gen. Relativ. Gravit. 33, 2101 (2001)

94. Y.C. Ong, JCAP 09, 015 (2018)

95. D.V. Fursaev, Phys. Rev. D 51, 5352 (1995)

96. R.B. Mann, S.N. Solodukhin, Nucl. Phys. B 523, 293 (1998)

97. R.K. Kaul, P. Majumdar, Phys. Rev. Lett. 84, 5255 (2000)

98. S. Kalyana Rama, Phys. Lett. B 566, 152 (2003)

99. D.N. Page, New J. Phys. 7, 203 (2005)

100. M. Domagala, J. Lewandowski, Class. Quantum Gravity 21, 5233 (2004)

101. J.D. Barrow, Phys. Lett. B 808, 135643 (2020)

102. E.M.C. Abreu, J.A. Neto, Phys. Lett. B 810, 135805 (2020) 